\title{
Variational and Shape Prior-based Level Set Model for Image Segmentation
}

\author{
El Hadji S. Diop ${ }^{\ddagger}$, Silèye O. Ba ${ }^{\dagger}$, Taha Jerbi ${ }^{\ddagger}$ and Valérie Burdin ${ }^{\ddagger}$ \\ † Image and Information Department, Telecom Bretagne / INSERM U650, Brest, France; \\ $\dagger$ Signal and Communications Department, Telecom Bretagne / Lab-STICC, Brest, France.
}

\begin{abstract}
A new image segmentation model based on level sets approach is presented herein. We deal with radiographic medical images where boundaries are not salient, and objects of interest have the same gray level as other structures in the image. Thus, an a priori information about the shape we look for is integrated in the level set evolution for good segmentation results. The proposed model also accounts a penalization term that forces the level set to be close to a signed distance function (SDF), which then avoids the re-initialization procedure. In addition, a variant and complete Mumford-Shah model is used in our functional; the added Hausdorff measure helps to better handle zones where boundaries are occluded or not salient. Finally, a weighted area term is added to the functional to make the level set drive rapidly to object's boundaries. The segmentation model is formulated in a variational framework, which, thanks to calculus of variations, yields to partial differential equations (PDEs) to guide the level set evolution. Results obtained on both synthetic and digital radiographs reconstruction (DRR) show that the proposed model improves on existing prior and non-prior shape based image segmentation.
\end{abstract}

Keywords: Image segmentation, level sets, shape prior, partial differential equations, calculus of variations.

PACS: 07.05.Pj, 87.57.nm, 02.30.Jr, 02.30.Xx

\section{INTRODUCTION}

Image segmentation still remains a very important task in image processing and computer vision fields. Although many applications based on image thresholding have been proposed, such an approach is definitely inefficient for accurate segmentation results. More elaborated methods such as active contours (AC) had then been introduced [1] by using dynamical curves to track objects boundaries. The parametrical formulation and implementation of AC was then improved by using level sets approach [2]. Represented as the zero level of a $2 D$ implicit function within an Eulerian framework, geometric AC (GAC) [3,4] improve greatly on parametric AC (PAC). Indeed, in addition to efficient numerical schemes, GAC allow topology changes conferring naturally breaking or merging during the evolution.

The evolution of $(\mathrm{P} / \mathrm{G}) \mathrm{AC}$ are guided by PDEs usually obtained through a minimization of a functional or energy defined in a way such that its minimum is reached at the object's boundary, which hold the highest image gradients. It is clear that if the object's boundary has low gradients, such methods will fail to properly segment desired features; this is particularly the case in most medical images. An alternative is to use an a priori information about the boundary so that the active contour or level set will also evolve according to a known shape for example. Such an approach was proposed in $[5,6,7,8]$ with different ways to create the prior training shape.

While the segmentation procedure remains crucial in many image processing applications, it is usually just a first step towards a target purpose. Extending previous works, we propose here a new segmentation method requiring less computation time and improving segmentation results, even if object boundaries are occluded, making the algorithm more robust than existing methods. The segmentation approach is detailed in the next section. In our medical application, it will be used as a pre-processing to perform a more robust and accurate $2 D / 3 D$ medical image registration. The $3 D$ object (long bone) is registrated on specific contours detected on DRR. On these projective gray level images, bone contours are not salient due to superimpositions and low contrast on local features.

\section{THE PROPOSED SEGMENTATION MODEL}

\section{Training shape design}

In this context a training shape is essential, and designed as in [5]. Let $\left\{\phi_{n}\right\}_{1 \leq n \leq N}$ be the set of $N$ aligned training curves embedded as zero level sets of higher dimension surfaces; embedding functions are truly SDFs [9]. Let 
$\bar{\phi}=\frac{1}{N} \sum_{n=1}^{N} \phi_{n}$ be the mean SDF; the shape variance is then computed by Principal Component Analysis (PCA) on $\left\{\phi_{n}\right\}_{1 \leq n \leq N}$. The new shape prior is given by $\phi=\bar{\phi}+U \lambda$, where $U=\left[u_{i}\right]_{1 \leq i \leq p}$ is the matrix of eigenvectors and $\lambda=\left[\lambda_{i}\right]_{1 \leq i \leq p}^{t \leq 1}$ is the vector of weights or shape parameters to be determined. Depending on the values of $\lambda_{i}$, the shape prior will be changed from the mean training set value $\bar{\phi}$ to fit the object boundary according to the level set evolution.

\section{New energy functional for image segmentation}

Let $I: \Omega \longrightarrow \mathbb{R}, \Omega \subset \mathbb{R}^{2}$ being an open and bounded set. Let us define the functional $F$ to minimize:

$F\left(\varphi, \lambda, V, I_{\text {in }}, I_{\text {out }}\right)=\frac{\alpha}{2} F_{1}(\varphi)+F_{2}(\varphi, \lambda, V)+\beta F_{3}(\varphi)+v F_{4}\left(\lambda, V, I_{\text {in }}, I_{\text {out }}\right)$,

where $\alpha, \beta$ and $v$ are positive weights to counterbalance effects of each term $F_{1}, F_{2}, F_{3}$ and $F_{4}$, detailed below.

The first internal energy $F_{1}$ is defined as: $F_{1}(\varphi)=\int_{\Omega}(|\nabla \varphi|-1)^{2} d x$.

$F_{1}$ depends only on $\varphi$, and maintains the evolving level set to be SDF. In fact, a SDF must satisfy the property of $|\nabla \varphi|=1$, and conversely, any function $\varphi$ satisfying $|\nabla \varphi|=1$ is a SDF plus a constant [10]. Such a functional was used in [11] to avoid the re-initialization process and its related drawbacks. To keep the level set as a SDF, the most common way is to solve the PDE: $\frac{\partial \varphi}{\partial t}=\operatorname{sign}\left(\varphi_{0}\right)(1-|\nabla \varphi|), \varphi_{0}$ being the function to be re-initialized.

We aim at segmenting objects of interest in images. So, we need to evolve the level set towards object boundaries, and define then $F_{2}$ such as: $F_{2}(\varphi, \lambda, V)=\int_{\Omega}\left[\xi g+\frac{\gamma}{2} \phi^{2}(\lambda, h(x))\right] \delta(\varphi)|\nabla \varphi| d x$,

where $\xi$ and $\gamma$ are positive weights, $\delta(\cdot)$ denotes the Dirac function, $g$ is the classical edge detector usually defined by $g=\frac{1}{1+\eta\left|\nabla G_{\sigma} \star I\right|^{2}}, \eta>0$ and $G_{\sigma}$ is a Gaussian kernel with standard deviation $\sigma$. We allow a spatial rigid transformation in the model carried out through a transformation vector $V=[\tau, \theta, T] \in \Omega_{V}$, with $\tau>0$ as a scale parameter, $\theta$ is a rotation angle and $T=\left[T_{x}, T_{y}\right]$ is a translation vector s.t. $h: \Omega \longrightarrow \Omega, h(x)=\tau R_{\theta} x+T$, where $R_{\theta}=\left(\begin{array}{cc}\cos \theta & \sin \theta \\ -\sin \theta & \cos \theta\end{array}\right)$.

The term $\phi^{2}(\lambda, h(x))$ in $F_{2}$ measures the similarity between a point $x \in \Omega$ of the zero level set and the new shape $\phi$ thanks to the shape parameters $\lambda$ and the eigenvectors $U$; a spatial transformation $h$ is allowed for a better fitting. $F_{2}$ was used in [12] by considering the training shape as the mean shape $\bar{\phi}$; it was also used in [13].

In our case as in many applications, the segmentation is only a first stage. Indeed, segmentation results will be used as a pre-processing in order to perform a $3 D$ registration. We propose then: $F_{3}(\varphi)=\int_{\Omega} g H(-\varphi) d x$,

with $H(\cdot)$ as the Heaviside function. $F_{3}$ can be interpreted as a weighted area of the target object to be segmented, and its minimization yields another force that quickly pushes the level set quickly towards the object boundaries.

Actually, we mainly deal with medical images for which objects of interest have a smooth gray level (see Figure 4). Therefore, to perform the segmentation, we finally define the last functional based on a modified Mumford-Shah functional [14], which is then the most suitable model for our images. Let $\Lambda_{i n}=\left|I-I_{\text {in }}\right|^{2}+\mu\left|\nabla I_{\text {in }}\right|^{2}, F_{4}$ is defined by: $F_{4}\left(\lambda, V, I_{\text {in }}, I_{\text {out }}\right)=\int_{\Omega} \Lambda_{\text {in }} H(\phi(\lambda, h(x))) d x+\int_{\Omega} \Lambda_{\text {out }}(1-H(\phi(\lambda, h(x))))+\zeta \int_{\Omega} d \mathscr{H}^{1}(C(\lambda, h(x))) d x$,

where $C(\lambda, h(x))=\{x \in \Omega$ s.t. $\phi(\lambda, h(x))=0\}$ and $\mathscr{H}^{1}$ is the Hausdorff measure. For instance, the first two terms of $F_{4}$ were proposed in [8]. As shown by results, the added Hausdorff measure has the benefit to account the length of the zero level of the new shape $\phi$ and helps to better handle edges when object's contours are occluded for example. The level set formulation of the proposed variational approach is then formulated as: $\min _{\varphi, \lambda, V, I_{\text {in }}, I_{\text {out }}} F\left(\varphi, \lambda, V, I_{\text {in }}, I_{\text {out }}\right)$. Evolution equations related to Euler-Lagrange equations of the functional $F$ are obtained by first variation descent with respect to $\varphi, I_{\text {in }}$ and $I_{\text {out }}$, and gradient descent with respect to $\lambda$ and $V$, as follows:

$$
\begin{gathered}
\left\{\begin{array}{c}
\frac{\partial \varphi}{\partial t}=\alpha\left(\triangle \varphi-\operatorname{div}\left(\frac{\nabla \varphi}{\mid \nabla \varphi}\right)\right)+\delta(\varphi) \operatorname{div}\left[(\xi g+\gamma \phi(\lambda, h(x))) \frac{\nabla \varphi}{|\nabla \varphi|}\right], \text { in } \Omega \times \mathbb{R}_{+}^{\star} \\
\frac{\partial \varphi}{\partial n}=0 \text { on } \partial \Omega \times \mathbb{R}_{+}^{\star} \text { and } \varphi(x, 0)=\varphi_{0}(x), x \in \Omega
\end{array}\right. \\
\left\{\begin{array}{r}
\frac{\partial \lambda}{\partial t}=-2 \gamma \int_{\Omega}\left(\nabla_{\lambda} \phi(\lambda, h(x))\right) \phi(\lambda, h(x)) \delta(\varphi)|\nabla \varphi| d x-v \int_{\Omega}\left(\Lambda_{\text {in }}-\Lambda_{\text {out }}\right) \nabla_{\lambda} \phi(\lambda, h(x)) \delta(\phi(\lambda, h(x))) d x \\
-\zeta \int_{\Omega} \nabla_{\lambda} \phi(\lambda, h(x)) \delta^{\prime}(\phi(\lambda, h(x))) d x, \text { in } \Omega_{\lambda} \times \mathbb{R}_{+}^{\star} \text { and } \lambda(x, 0)=\lambda_{0} \text { in } \Omega_{\lambda}
\end{array}\right. \\
\left\{\begin{array}{r}
\frac{\partial V}{\partial t}=-2 \gamma \int_{\Omega}\left(\nabla_{V} h(x)\right)(\nabla \phi(\lambda, h(x))) \phi(\lambda, h(x)) \delta(\varphi)|\nabla \varphi| d x-v \int_{\Omega}\left(\Lambda_{\text {in }}-\Lambda_{\text {out }}\right) \nabla_{\lambda} \phi(\lambda, h(x)) \delta(\phi(\lambda, h(x))) d x \\
-\zeta \int_{\Omega}\left(\nabla_{V} h(x)\right)(\nabla \phi(\lambda, h(x))) \delta^{\prime}(\phi(\lambda, h(x)))+\left\langle\nabla_{V}(\nabla \phi(\lambda, h(x))), \frac{\nabla \phi(\lambda, h(x))}{|\nabla \phi(\lambda, h(x))|}\right\rangle \delta(\phi) d x, \text { in } \Omega_{V} \times \mathbb{R}_{+}^{\star} \text { and } V(x, 0)=V_{0} \text { in } \Omega_{V}
\end{array}\right.
\end{gathered}
$$




$$
\left\{\begin{array}{c}
\frac{\partial I_{\text {out }}}{\partial t}=I-I_{\text {out }}+\mu \triangle I_{\text {out }} \text { in } \Omega_{\text {out }}=\{x, \phi(\lambda, h(x))<0\} \times \mathbb{R}_{+}^{\star} \text { and } \frac{\partial I_{\text {in }}}{\partial t}=I-I_{\text {in }}+\mu \triangle I_{\text {in }}, \text { in } \Omega_{\text {in }}=\{x, \phi(\lambda, h(x))>0\} \times \mathbb{R}_{+}^{\star} \\
\frac{\partial I_{\text {out }}}{\partial n}=0 \text { on }\left(\partial \Omega_{\text {out }} \cup\{x \in \Omega \text { s.t. } \phi(\lambda, h(x))=0\}\right) \times \mathbb{R}_{+}^{\star}, I_{\text {out }}(x, 0)=I(x), x \in \Omega_{\text {out }} \\
\frac{\partial I_{\text {in }}}{\partial n}=0 \text { on }\left(\partial \Omega_{\text {in }} \cup\{x \in \Omega \text { s.t. } \phi(\lambda, h(x))=0\}\right) \times \mathbb{R}_{+}^{\star} \text { and } I_{\text {in }}(x, 0)=I(x), x \in \Omega_{\text {in }}
\end{array}\right.
$$

RESULTS

The segmentation model guided by equations (4), (3), (2) and (1) is implemented in this order. As in [15], slightly regularized versions of $\delta, \delta^{\prime}$ and $H$ are used instead, and the term $|\nabla \varphi|$ is computed with the Fast Marching algorithm [2]. The model gives satisfactory results on simple synthetic images, as well as the approach in [8]. Aiming at segmenting real objects for which boundaries are not salient, it would be better to apply the algorithm on more realistic synthetic images. Therefore, the model is also validated on a synthetic image with partial occlusion on boundaries (cf. Figure 2-(a)) to simulate superimposed shapes; the training set is composed of $N=30$ shapes. Figure 1 illustrates the set of aligned contours and new created shapes based on the mean shape value and the PCA, which provides two principal components with a fitting percentage of $98 \%$. Figure 2 shows the segmentation results obtained with the

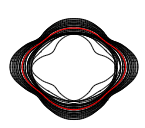

(a)

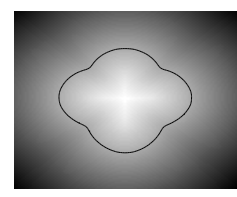

$\bar{\phi}+3 U_{1}$

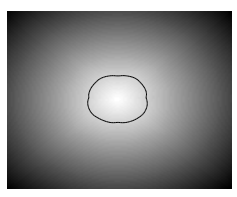

$\bar{\phi}-3 U_{1}$

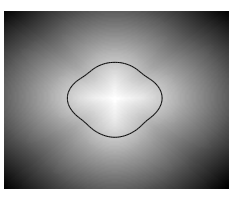

Mean value $\bar{\phi}$

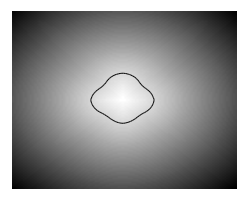

$\bar{\phi}+3 U_{2}$

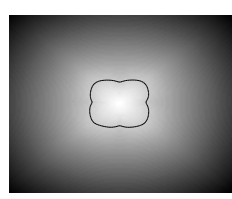

$\bar{\phi}-3 U_{2}$

FIGURE 1. New created shapes based on shape parameters, PCA and mean SDFs; zero level sets of new shapes are plotted in dark. Figure (a) represents the aligned curves of the training dataset; the mean shape is plotted in red.

proposed model, approach presented in [8] and the non shape prior-based segmentation proposed in [11]. As expected, the latter one does not stop on true object boundaries; because, on the only basis of the image gradients, the considered object will be like the whole shape including the rectangle that serves to create the occlusion. On the other hand, with the same parameters as in our approach, object boundaries are well handled with [8] except at regions where the occlusion takes place, despite the 40 iterations needed to reach the stationary solution, while just 11 iterations are necessary with our approach. This example illustrates the benefits, in the minimization process, of the added $F_{1}$ and $F_{3}$ functionals, and the Hausdorff measure in $F_{4}$. Indeed, $F_{3}$ makes the active contour evolve more rapidly to object boundaries, and the Hausdorff measure constraints it to be as close as possible to the shape prior, meanwhile keeping a minimal length. Finally, $F_{1}$ maintains the active contour to be a SDF.

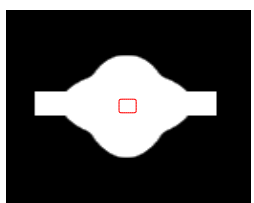

(a)

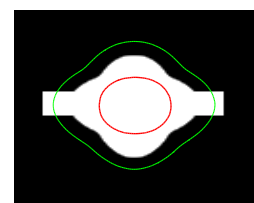

(b)

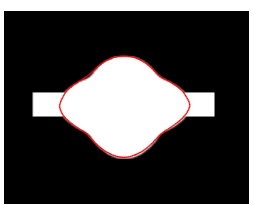

(c)

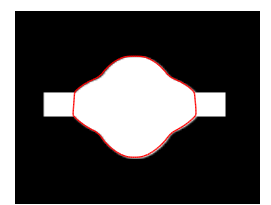

(d)

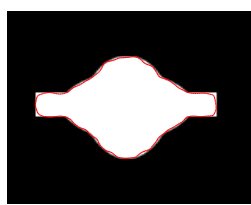

(e)

FIGURE 2. (a) Level set initialization in red. (b) Level set evolution in red and shape prior in green, with the proposed approach. Final segmentation with: (c) Our approach, (d) Approach proposed in [8] and (e) Segmentation without shape prior [11].

For the DRR segmentation, we need to approach the bone variability by using a training dataset composed of simulated X-rays projections with variable angles range between $\left[\theta-10^{\circ}, \theta+10^{\circ}\right]$, where $\theta$ is a choosen projection angle regards the longitudinal bone axis. The first step consists in aligning the training contours $\left\{C_{n}\right\}_{1 \leq n \leq N}$ to $C_{1}$ (one could choose another one) by finding $\forall n \in \llbracket 2, n \rrbracket$ the scale $s_{n}$, angle $\theta_{n}$ and translation $T_{n}=\left[T_{x}^{n}, T_{y}^{n}\right]$ that minimize the measure [16]: $m\left(C_{1}, C_{n}^{\mathrm{al}}\right)=$ area of $\left(A_{1} \cup A_{n}^{\mathrm{al}}-A_{1} \cap A_{n}^{\mathrm{al}}\right)$, where $A_{n}$ is the area enclosed by the curve $C_{n}$, and $C_{n}^{a l}=s_{n} R_{\theta_{n}} C_{n}+T_{n}$.

The PCA is then applied on SDFs of 16 aligned bone contours, which gives 3 principal components for a fitting percentage of $86 \%$ (see Figure 3). Segmentation results are displayed in Figure 4 showing a better bone segmentation with our approach for the same reasons previously discussed for the synthetic image. 


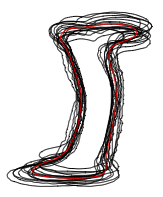

(a)

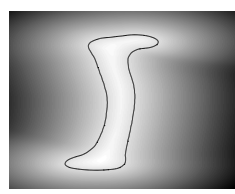

$\bar{\phi}+0.9 U_{1}$

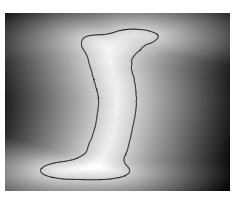

$\bar{\phi}+0.9 U_{2}$

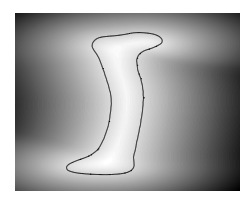

Mean value $\bar{\phi}$

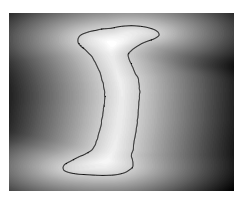

$\bar{\phi}-0.9 U_{3}$

FIGURE 3. (a) Aligned training bone contours; mean shape is plotted in red.

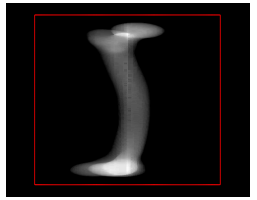

Initialization on DRR

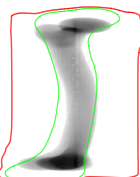

Evolution

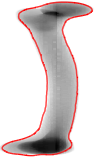

Our approach

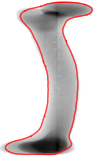

Approach in [8]

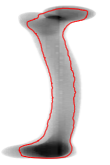

Without shape prior [11]

FIGURE 4. Segmentation results on DRR

\section{CONCLUSION AND PERSPECTIVES}

A new image segmentation model based on level sets approach is presented in this paper. Formulated in a variational framework, the proposed functional is composed of four terms. The first one keeps the level set to be close to a SDF. The second term incorporates in the level set evolution an a priori information about shapes we look for. The third one is a weighted area term to make the level set drive rapidly to object's boundaries. The last term is a variant and complete Mumford-Shah functional. Preliminary results obtained on both synthetic and DRR images show that the proposed approach improves on prior and non-prior based image segmentation. As for future works, the proposed model will be applied on true medical radiographs with different structures in images (bones, soft tissues, $\cdots$ ). Also, a theoretical study of the minimum of the proposed functional will be conducted.

\section{ACKNOWLEDGMENTS}

Authors are grateful to Fred Ngolemboula for helping design the bone training contours during his summer engineering internship in the Image and Information Processing Department, Telecom Bretagne.

\section{REFERENCES}

1. M. Kass, A. Witkin, and D. Terzopoulos, International Journal of Computer Vision 1988, 321-331 (1987).

2. S. Osher, and J. A. Sethian, Journal of Computational Physics 79, 12-49 (1988).

3. R. Malladi, J. A. Sethian, and B. C. Vemuri, IEEE Trans. on Pattern Analysis and Machine Intelligence 17, 158-175 (1995).

4. V. Caselles, R. Kimmel, and G. Sapiro, International Journal of Computer Vision 22, 61-79 (1997).

5. M. E. Leventon, W. E. L. Grimson, and O. Faugeras, "Statistical Shape Influence in Geodesic Active Contours," in CVPR, South Carolina, USA, 2000, pp. 316-323.

6. D. Cremers, T. Kohlberger, and C. Schnörr, "Nonlinear shape statistics in Mumford-Shah based segmentation,", in ECCV, Springer, Copenhagen, Denmark, 2002, vol. 2351, pp. 93-108.

7. M. Rousson, and N. Paragios, "Shape Priors for Level Set Representations," in ECCV, Copenhagen, 2002, pp. 78-92.

8. X. Bresson, P. Vandergheynst, and J.-P. Thiran, International Journal of Computer Vision 68, 145-162 (2006).

9. S. Osher, and R. Fedkiw, Level Set Methods and Dynamic Implicit Surfaces, Applied Mathematical Sciences, Springer, 2003.

10. V. I. Arnold, Geometrical Methods in the Theory of Ordinary Differential Equations, Springer-Verlag, 1983.

11. C. Li, C. Xu, C. Gui, and M. D. Fox, "Level Set Evolution Without Re-initialization: A New Variational Formulation," in IEEE CVPR, Washington DC, USA, 2005, vol. 1, pp. 430-436.

12. Y. Chen, H. D. Tagare, S. Thiruvenkadam, F. Huang, D. Wilson, K. S. Gopinath, R. W. Briggs, and E. A. Geiser, International Journal of Computer Vision 50, 315-328 (2002).

13. X. Bresson, P. Vandergheynst, and J.-P. Thiran, "A Priori Information in Image Segmentation: Energy Functional based on Shape Statistical Model and Image Information," in IEEE ICIP, Barcelona, Spain, 2003, vol. 3, pp. 428-428.

14. D. Mumford, and J. Shah, Communications on Pure and Applied Mathematics 42, 577-685 (1989).

15. H.-K. Zhao, T. Chan, B. Merriman, and S. Osher, Journal of Computational Physics 127, 179-195 (1996).

16. R. Veltkamp, and M. Hagedoorn, State-of-the-Art in Shape Matching, Tech. Rep. UU-CS-1999-27, Utrecht University (1999). 